\title{
Biocombustible Sólido A Partir De Residuos Que Generan Los Procesos Agroindustriales Del Sector El Empalme
}

SOLID BIOFUEL FROM WASTE GENERATED BY AGROINDUSTRIAL PROCESSES IN THE EL EMPALME SECTOR

\author{
José Villarroel Bastidas ${ }^{1}$ \\ Universidad Técnica Estatal de Quevedo \\ Josselin Macías Vera ${ }^{2}$ \\ Universidad Técnica Estatal de Quevedo \\ Bryan Espinoza Oviedo ${ }^{*}$ \\ Universidad Técnica Estatal de Quevedo
}

Fecha recepción: 15 de junio de 2020

Fecha aceptación: 16 de julio de 2020

(1) 2020 Universidad de Cordoba. Este es un artículo de acceso abierto distribuido bajo los términos de la licencia Creative Commons Attribution License, que permite el uso ilimitado, distribución y reproducción en cualquier medio, siempre que el autor original y la fuente se acreditan.

\section{RESUMEN}

El biocombustible sólido, se obtiene mediante la compactación o densificación de residuos, no contienen aglutinantes, aditivos ni otra sustancia que puedan deteriorar las calderas e instalaciones de calefacción, su contenido es $100 \%$ residuos de los procesos

\section{ABSTRACT}

Solid biofuel is obtained by compacting or densifying waste, it does not contain binders, additives or other substances that can deteriorate boilers and heating installations, its content is $100 \%$ waste from agro-industrial processes, lignin acts as a natural binder. The

1 Facultad de Ciencias de la Ingeniería, Quevedo, Ecuador jvillarroel @uteq.edu.ec, https://orcid.org/0000-0002-9013-37763

2 Ingeniera Agroindustrial, Facultad de Ciencias de la Ingeniería, Quevedo, Ecuador josselinmver.macias@uteq.edu.ec

3 Bryan Espinoza Oviedo, Ingeniero Agroindustrial, Unidad Educativa Alec Peterson, Milagro 
agroindustriales, la lignina actúa como aglutinante natural. Como objetivo principal es la preparación y caracterización física y química de la briqueta para ser utilizadas como biocombustible sólido, a los residuos Agroindustriales como: cascarilla de arroz, polvillo, cuesco y aserrín. Con un diseño de bloques completamente al azar y un arreglo factorial $\mathrm{ABC}$ que constó de 12 tratamientos y 2 réplicas lo que corresponde a 24 tratamientos. Los factores de estudio: Factor A (tipo de mezcla), Factor $B$ (forma de las briquetas) y Factor C (tamaño de las briquetas). Para el proceso de compactación se diseñó y fabricó moldes de formas cilíndricas y rectangulares de 10 y $15 \mathrm{~cm}$ de altura. Se determinó el mejor tratamiento mediante análisis físicos y químicos preparado con cáscara de arroz $4 \%$, polvillo $14 \%$, cuesco 10 $\%$, Aserrín $12 \%$, de forma cuadrada con un tamaño de $10 \mathrm{~cm}$, con las siguientes características: $4.35 \%$ cenizas, $11 \%$ de humedad, poder calorífico $5268.20 \mathrm{Kcal} / \mathrm{kg}$, densidad $1 \mathrm{~g} / \mathrm{cm}^{3}$ y una fuerza de compactación de 6.51 PSI; al ser este el tratamiento sobresaliente con las características deseadas en una briqueta, se procedió a realizar pruebas de temperatura y tiempo de combustión, donde alcanzo un rango de (127- 240$)^{\circ} \mathrm{C}$ en un tiempo de 30 minutos para ser totalmente calcinada.

PALABRAS CLAVE: Poder calorífico, biomasa, Compactación, energía, combustión. main objective is the preparation and physical and chemical characterization of the briquette to be used as a solid biofuel, to Agroindustrial residues such as: rice husk, dust, stone and sawdust. With a completely randomized block design and an $\mathrm{ABC}$ factorial arrangement that consisted of 12 treatments and 2 replicates, which corresponds to 24 treatments. The study factors: Factor A (type of mixture), Factor B (shape of the briquettes) and Factor $C$ (size of the briquettes). For the compaction process, molds were designed and manufactured with cylindrical and rectangular shapes of 10 and $15 \mathrm{~cm}$ in height. The best treatment was determined by physical and chemical analysis prepared with $4 \%$ rice husk, $14 \%$ dust, $10 \%$ husk, $12 \%$ sawdust, square in shape with a size of $10 \mathrm{~cm}$, with the following characteristics: $4.35 \%$ ash, $11 \%$ humidity, calorific value $5268.20 \mathrm{Kcal} / \mathrm{kg}$, density $1 \mathrm{~g} /$ $\mathrm{cm}^{3}$ and a compaction force of 6.51 PSI; This being the outstanding treatment with the desired characteristics in a briquette, we proceeded to carry out temperature and combustion time tests, where it reached a range of $(127-240){ }^{\circ} \mathrm{C}$ in a time of 30 minutes to be fully calcined.

KEYWORDS: Calorific power, biomass, Compaction, energy, combustion. 


\section{INTRODUCCIÓN}

Esta investigación tiene como fin aprovechar y dar un valor agregado a los residuos Agroindustriales que se generan en grandes cantidades en la zona del cantón El Empalme Provincia del Guayas por la actividad agrícola y forestal que no son aprovechadas $\mathrm{o}$ industrializadas debido a la falta de información de estos subproductos que tienen un gran potencial para ser reutilizados en la elaboración de briquetas.

En la actualidad la utilización de estos subproductos tienen un alto grado de desaprovechamiento, debido a que se almacenan en grandes espacios físicos o se queman en calderas, sin poseer un mayor valor agregado o alcanzar una eficiencia energética mayor; unas de las vías para utilizar los residuos es convirtiéndolos en biocombustibles sólidos densificados ya que al fabricar y comercializar este tipo de combustibles, se disminuye considerablemente la cantidad de residuos, se reduce el volumen transportado, así como también se logra una combustión más limpia y eficiente (Gallipoliti, V; Martina, P; Corace , J; Aeberhardt, R; Garcia, Sola, 2012).

Las briquetas son utilizadas para generar calor utilizados en estufas, chimeneas, salamandras, hornos y calderas; es un producto $100 \%$ ecológico y renovable, catalogada como bio-energía sólida, que viene en forma cilíndrica o de ladrillo y sustituye a la leña con muchas ventajas: poder calorífico similar, fácil y rápido encendido, baja humedad, alta densidad, ocupa menos espacio, homogéneas, fácil manipulación, sin olores, humos ni chispas y menor porcentajes de cenizas (Gallipoliti, V; Martina, P; Corace , J; Aeberhardt, R; Garcia, Sola, 2012).

El objetivo de esta investigación fue la preparación y caracterización física y química de las briquetas con el fin de establecer la combinación óptima entre cascarilla de arroz, polvillo, aserrín y cuesco de Palma Africana, utilizando diferentes mezclas como material aglomerante, donde se realizó estudios de eficiencia energética (poder calorífico), contenido de humedad, ceniza, densidad, tamaño (dimensiones) y su forma.

\section{MARCO TEÓRICO O REFERENCIAL}

\subsection{Biocombustibles sólidos}

Son biocombustibles sólidos aquellos productos derivados de la biomasa sólida, los más importantes son los del tipo primario, constituidos por materias lignocelulósicas procedentes del sector agrícola y forestal y de las industrias de transformación que producen residuos de dicha naturaleza; la paja y los restos de poda de vid, olivo y frutales, la leña, las cortezas y los restos de podas y aclareos de las masas forestales son materias típicas para la elaboración de biocombustibles sólidos de origen agrario o forestal (Mosquera \& Merino, 2006).

\subsection{Briquetas}

Se obtienen mediante la compactación o densificación de residuos (de origen lignocelulósico u otros materiales) y se producen bajo la aplicación de grandes presiones y temperaturas elevadas que 


\section{RIIN}

provocan la autoaglomeración de sus partículas, o mediante bajas y medianas presiones con ayuda de una sustancia aglomerante para lograr su compactación (Da Silva, 2013)

\subsection{Densidad de las briquetas}

Los factores que influyen en la densidad son la materia prima empleada y la presión ejercida por la prensa, cuanto mayor sea la densidad de la materia prima mayor será la densidad del producto final donde la presión de compactación en el proceso de fabricación son variables, y depende únicamente de la maquinaria utilizada (Da Silva, 2013).

\subsection{Humedad de las briquetas}

Para obtener productos compactados de calidad es preciso que el contenido de humedad no supere valores del orden del $15 \%$ y que esté por encima de valores del $8 \%$, puesto que por debajo de este nivel no se obtienen una adecuada aglomeración, ya que el agua en determinadas proporciones actúa como sustancia termoplástica favoreciendo la autoglomeración y, por tanto, si no alcanzan estos porcentajes mínimos la compactación es defectuosa (Ortiz, 2008).

\subsection{Contenido de cenizas}

Los valores típicos de las cenizas o residuos sólidos no quemados varían entre 2 y $5 \%$, cuando es superior al $15 \%$ incide negativamente en el rendimiento energético debido a que no es posible el aprovechamiento de la energía útil y genera problemas de sinterización. [10] Los valores de la humedad libre superficial y la retenida en los poros de la biomasa, están alrededor del $8 \%$ al $50 \%$ y para la materia volátil, el valor es mayor al $65 \%$ (Muñoz Muñoz, Pantoja, Alvaro, Cuatin,
\& F., 2014).

\subsection{Poder Calorífico}

Es la cantidad de calor liberado durante la combustión por unidad de masa, a presión constante y a $25^{\circ} \mathrm{C}$, según el estado de los productos, el Poder Calorífico Superior (P.C.S.) incluye el calor latente, mientras el Poder Calorífico Inferior (P.C.I.), no lo considera, la biomasa tiene un poder calorífico $50 \%$ menor que el resto de los combustibles, excepto al carbón (Muñoz Muñoz, Pantoja, Alvaro, Cuatin, \& F., 2014).

Una de las características principales en el biocombustible sólido es su poder calorífico ya que depende de la composición química del mismo, el cual se define como tal, altos poderes caloríficos indican buenos combustibles y bajos poderes caloríficos señalan malos combustibles [15]. Para el caso de las briquetas, el poder calorífico está en función del material del cual está compuesto, suponiendo que la briqueta está compuesta de madera sin aditivos, entonces su poder calorífico será igual al de la madera que la conforma, análisis realizados en las briquetas indican que el poder calorífico de las briquetas es mayor a $4300 \mathrm{kcal} / \mathrm{kg}$ (García, 2014).

\section{METODOLOGÍA DE LA INVESTIGACIÓN}

Los tratamientos se realizaron en el taller mecánico de la Universidad Técnica Estatal de Quevedo (UTEQ), situado en el $\mathrm{km} 7$ de la Vía Quevedo - Santo Domingo, Cantón Quevedo, Provincia de Los Ríos, entre las coordenadas geográficas $01^{\circ} 06^{\prime}$ 
de latitud Sur y $79^{\circ} 29^{\prime}$ de longitud Oeste. A una altura de $73 \mathrm{msnm}$. Los diferentes residuos Agroindustriales como (cascarilla de arroz, cuesco, aserrín, y polvillo) para la elaboración de las briquetas, fueron recolectados en distintas empresas ubicadas en el sector "El Empalme" provincia del Guayas.

Los análisis de las briquetas fueron realizados en el laboratorio de bromatología del campus finca experimental La María de la UTEQ, localizado en el $\mathrm{km} 7$ de la vía Quevedo El Empalme, C. P. 73. Mocache, Los Ríos. Situada en las coordenadas geográficas $70^{\circ} 27^{\prime} 13$ " de latitud Sur y 0106'02”' de longitud Oeste, a una altura de $73 \mathrm{msnm}$, temperatura promedio de $25^{\circ} \mathrm{C}$, humedad relativa de $84.0 \%$ la cual cuenta con los equipos y materiales necesarios para la realización de los análisis de humedad, densidad aparente, cenizas y poder calorífico.

\subsection{Diseño de la investigación}

Se utilizó el diseño completamente al azar DCA para establecer la combinación adecuada en la elaboración de briquetas, procesándose los datos a través de un ANOVA de tres factores teniendo como tratamientos la mezcla, forma y tamaño, luego se empleó la prueba de TUKEY $(\mathrm{p} \leq 0.05)$ para determinar la diferencia entre tratamientos.

Para efectuar la investigación se empleó los siguientes factores de estudios:

A= Tipo de mezcla (cascarilla de arroz - polvillo - cuesco - aserrín).

B= La forma (cilíndrica - cuadrada)

C= Tamaño $(10 \mathrm{~cm}-15 \mathrm{~cm})$.

Tabla 1. Factores de estudio que intervienen en la elaboración de briqueta.

\begin{tabular}{|c|c|c|}
\hline FACTORES DE ESTUDIO & SIMBOLOGÍA & DESCRIPCIÓN \\
\hline \multirow{18}{*}{ Factor A: Mezcla. } & \multirow{6}{*}{$a_{0}$} & Mezcla 1 \\
\hline & & Cáscara de arroz $1 \%$ \\
\hline & & Polvillo $16 \%$ \\
\hline & & Cuesco $8 \%$ \\
\hline & & Aserrín $15 \%$ \\
\hline & & Agua $\quad 60 \%$ \\
\hline & \multirow{6}{*}{$a_{1}$} & Mezcla 2 \\
\hline & & Cáscara de arroz $2 \%$ \\
\hline & & Polvillo $14 \%$ \\
\hline & & Cuesco $12 \%$ \\
\hline & & Aserrín $12 \%$ \\
\hline & & Agua $\quad 60 \%$ \\
\hline & \multirow{6}{*}{$a_{2}$} & Mezcla 3 \\
\hline & & Cáscara de arroz $4 \%$ \\
\hline & & Polvillo $14 \%$ \\
\hline & & Cuesco $10 \%$ \\
\hline & & Aserrín $12 \%$ \\
\hline & & Agua $\quad 60 \%$ \\
\hline \multirow[t]{2}{*}{ Factor B: Forma. } & $b_{0}$ & Cilíndrica \\
\hline & $b_{1}$ & Cuadrada \\
\hline \multirow[t]{2}{*}{ Factor C: Tamaño. } & $c_{0}$ & $10 \mathrm{~cm}$ \\
\hline & $c_{1}$ & $15 \mathrm{~cm}$ \\
\hline
\end{tabular}


Elaborado por: Villarroel, J. \& Macías. J. 2020

Tabla 2. Combinación de los tratamientos propuesto para la elaboración de briquetas.

Tratamientos

$\begin{array}{ll}\text { T1 } & a_{0} b_{0} c_{0} \\ \text { T2 } & a_{0} b_{0} c_{1} \\ \text { T3 } & a_{0} b_{1} c_{0} \\ \text { T4 } & a_{0} b_{1} c_{1} \\ \text { T5 } & a_{1} b_{0} c_{0} \\ \text { T6 } & a_{1} b_{0} c_{1} \\ \text { T7 } & a_{1} b_{1} c_{0} \\ \text { T8 } & a_{1} b_{1} c_{1} \\ \text { T9 } & a_{2} b_{0} c_{0} \\ \text { T10 } & a_{2} b_{0} c_{1} \\ \text { T11 } & a_{2} b_{1} c_{0} \\ \text { T12 } & a_{2} b_{1} c_{1}\end{array}$

Descripción

$$
\begin{aligned}
& \text { Mezcla } 1+\text { cilíndrica }+10 \mathrm{~cm} \\
& \text { Mezcla } 1+\text { cilíndrica }+15 \mathrm{~cm} \\
& \text { Mezcla } 1+\text { cuadrada }+10 \mathrm{~cm} \\
& \text { Mezcla } 1+\text { cuadrada }+15 \mathrm{~cm} \\
& \text { Mezcla } 2+\text { cilíndrica }+10 \mathrm{~cm} \\
& \text { Mezcla } 2+\text { cilíndrica }+15 \mathrm{~cm} \\
& \text { Mezcla } 2+\text { cuadrada }+10 \mathrm{~cm} \\
& \text { Mezcla } 2+\text { cuadrada }+15 \mathrm{~cm} \\
& \text { Mezcla } 3+\text { cilíndrica }+10 \mathrm{~cm} \\
& \text { Mezcla } 3+\text { cilíndrica }+15 \mathrm{~cm} \\
& \text { Mezcla } 3+\text { cuadrada }+10 \mathrm{~cm} \\
& \text { Mezcla } 3+\text { cuadrada }+15 \mathrm{~cm}
\end{aligned}
$$

Para el proceso de compactación se diseñó y se fabricó moldes con alturas de $10 \mathrm{~cm}$ y $15 \mathrm{~cm}$ de forma rectangular y cuadrada, como se muestra en el gráfico 1, los cuales garantizarón la solidez del material al momento de extraer el molde. Se lo realizó utilizando una prensa hidráulica de 15 toneladas fuerzas en la cual se prensó el material dentro del molde eliminando el mayor porcentaje de agua.

El secado de las briquetas se efectuó utilizando una mufla ajustada a una temperatura de $50{ }^{\circ} \mathrm{C}$ por 24 horas, transcurrido el tiempo se retiró las briquetas y se dejó a la intemperie con intenso sol, hasta obtener un peso constante para conseguir briquetas con menor porcentaje de humedad para su fácil combustión. Cuando las briquetas alcanzaron un peso constante fueron almacenadas en un lugar seco libre de humedad, para luego ser sometida a los diferentes análisis establecidos en la investigación. 


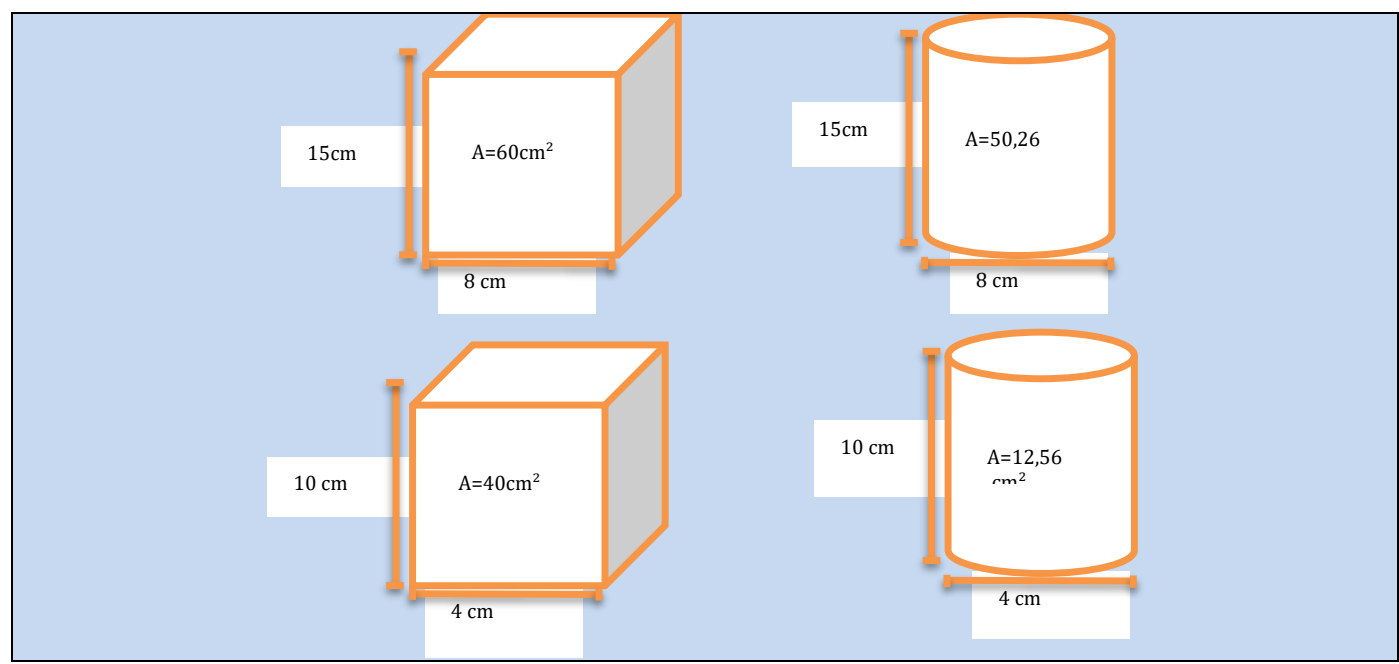

Gráfico 1. Diseño de moldes para el proceso de elaboración de las briquetas Elaborado por: Villarroel, J. \& Macías. J. 2020

\section{RESULTADOS}

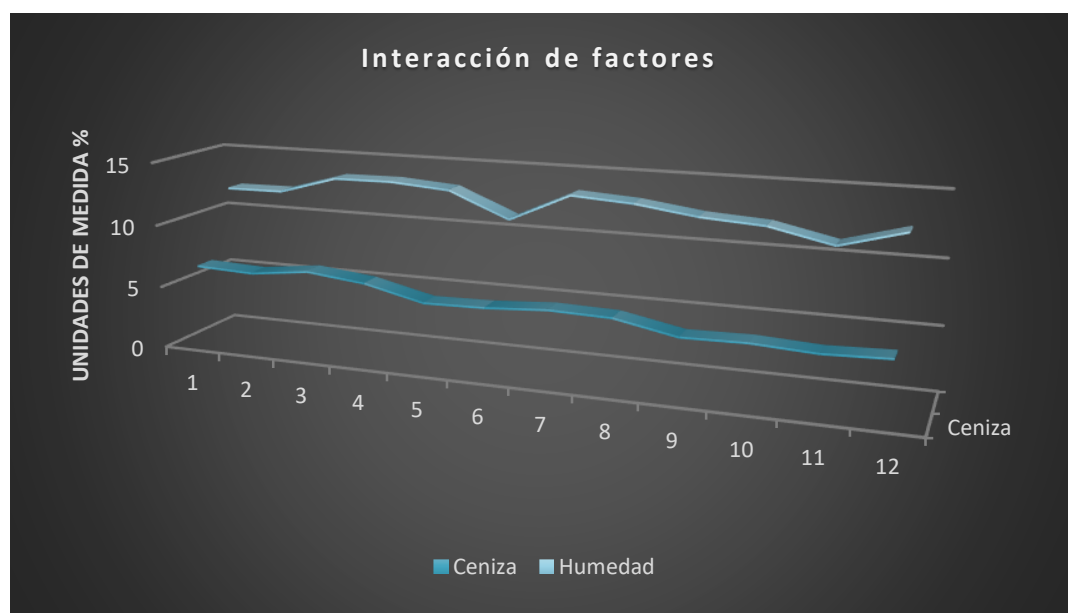

Gráfico 2. Muestra los resultados de la prueba de Tukey $(\mathrm{p}<0.05)$ de la interacción de $\mathrm{A}^{*} \mathrm{~B}^{*} \mathrm{C}$.

Diferencias de medias entre tamaño de las briquetas de la prueba de significación Tukey $(\mathrm{p}<0.05)$. 1.- Ceniza (DS); 2.-Humedad (DS) Elaborado por: Villarroel, J. \& Macías. J. 2020

El gráfico $\mathrm{N}^{\circ} 2$, indica el resultado de la interacción $\mathrm{A} \times \mathrm{B} \times \mathrm{C}$ en Ceniza el cual presentó diferencia significativa con valores bajos en las interacciones $a 0+b 0$ $+c 0=($ Mezcla $1+$ cilíndrica $+10 \mathrm{~cm})$, $a 0+b 0+c 1$ (Mezcla $1+$ cilíndrica +15 $\mathrm{cm}), a 0+b 1+c 0=($ Mezcla $1+$ cuadrada $+10 \mathrm{~cm})$, mientras que los más altos los reportaron $a 2+b 0+c 0=($ Mezcla $3+$ cilíndrica $+10 \mathrm{~cm}), a 2+b 0+c 1=$ 
(Mezcla $3+$ cilíndrica $+15 \mathrm{~cm} \mathrm{4),} \mathrm{a2+}$ $b 1+c 0=$ (Mezcla $3+$ cuadrada $+10 \mathrm{~cm}$ 3), $a 2+b 1+c 1=$ (Mezcla $3+$ cuadrada $+15 \mathrm{~cm}$ ).

Con respecto a la humedad el resultado de la interacción $\mathrm{A} \times \mathrm{B} \times \mathrm{C}$, presentó diferencia significativa con valores bajos en las interacciones $a 0+b 1+c 1=$ (Mezcla $1+$ cuadrada $+15 \mathrm{~cm} \mathrm{4),a0+b1+c0=}$
(Mezcla $1+$ cuadrada $+10 \mathrm{~cm}), a 1+b 1$ $+c 0=($ Mezcla $2+$ cuadrada $+10 \mathrm{~cm})$, $a 1+b 0+c 0=($ Mezcla $2+$ cilíndrica + $10 \mathrm{~cm}$ ), $a 1+b 1+c 1=$ (Mezcla $2+$ cuadrada $+15 \mathrm{~cm})$, mientras que los más altos los reportaron $a 1+b 0+c 1=$ (Mezcla $2+$ cilíndrica $+15 \mathrm{~cm}), a 2+b 1$ $+c 0=$ (Mezcla $3+$ cuadrada $+10 \mathrm{~cm})$.

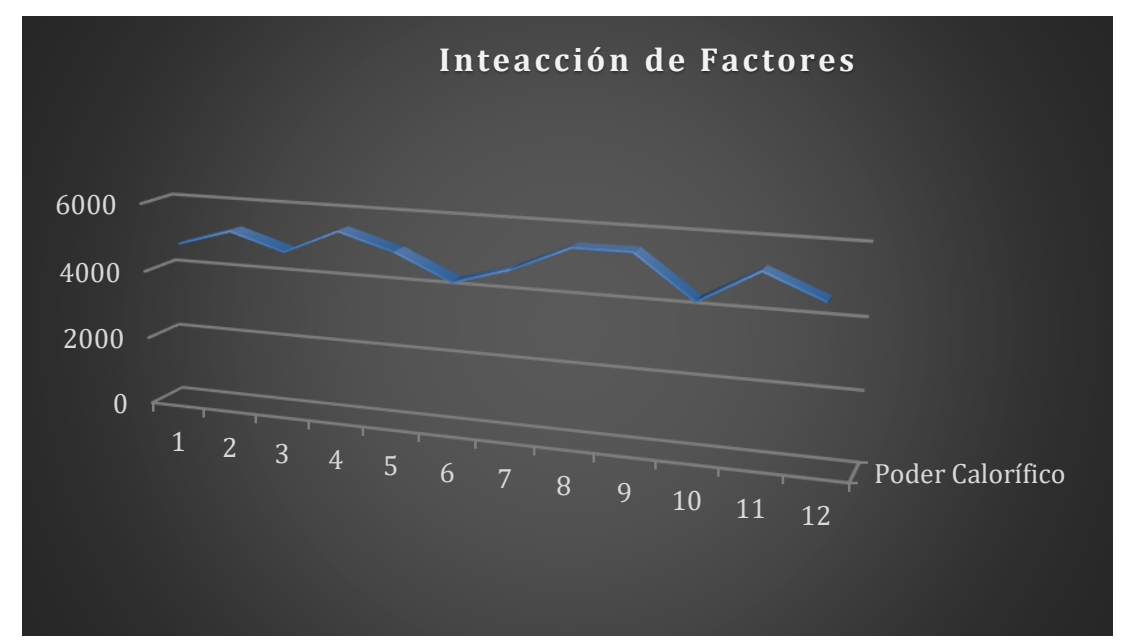

Gráfico 3. Muestra los resultados de la prueba de Tukey $(\mathrm{p}<0.05)$ de la interacción de $\mathrm{A}^{*} \mathrm{~B}^{*} \mathrm{C}$. Diferencias de medias de las briquetas de la prueba de significación Tukey $(\mathrm{p}<0.05)$. Poder calorífico (DS) Elaborado por: Villarroel, J. \& Macías. J. 2020

El gráfico $\mathrm{N}^{\circ} 3$, indica el resultado de la interacción $\mathrm{A} \times \mathrm{B} \times \mathrm{C}$ del contenido calorífico el cual presentó diferencia significativa con valores bajos en las interacciones $a 2+b 0+c 0=$ (Mezcla $3+$ cilíndrica $+10 \mathrm{~cm}), a 1+b 1+c 1=$ (Mezcla $2+$ cuadrada $+15 \mathrm{~cm}$ ), mientras que los más altos los reportaron $a 2+b 0$ $+c 1=($ Mezcla $3+$ cilíndrica $+15 \mathrm{~cm})$, $a 1+b 0+c 1=($ Mezcla $2+$ cilíndrica + $15 \mathrm{~cm})$.

\section{Discusión}

\subsection{Cenizas}

En relación a ceniza se obtuvo un valor de $4.69 \%$ para la mezcla 3 (cascarilla $4 \%$, polvillo $14 \%$, cuesco $10 \%$ y aserrín $14 \%$ ), este valor es similar al reportado por los autores (Muñoz Muñoz, Pantoja, Alvaro, Cuatin, \& F., 2014), En su investigación sobre Aprovechamiento de los residuos agroindustriales como biocombustible y biorrefinería, la cual indica que el porcentaje obtenido de cenizas en su estudio es de $4,12 \%$.

Además se hallaron valores de $5.53 \%$ para la forma cuadrada y $5.66 \%$ en la forma cilíndrica, estos datos están por debajo de $(8,13,20) \%$ de cenizas referenciados por (Valderrama, Curo, Quispe, Llantoy, \& Gallo, 2014) en su investigación con el tema, "Briquetas de residuos sólidos orgánicos como fuente de 
energía calorífica en cocinas no convencionales", lo cual indica que utilizar diferente formas para la elaboración de las briquetas no afecta en el contenido de cenizas del biocombustible, sin embargo utilizar formas cilíndricas ayudan a no desintegrarse con los golpes, a diferencia de las briquetas de formas cuadradas que se desintegran con gran facilidad al ser manipuladas (Valderrama, Curo, Quispe, Llantoy, \& Gallo, 2014).

El autor (Fernández Llorente, 2011) "En su estudio con el tema Características, barreras y retos para la implantación comercial de la biomasa sólida como combustible", indica que el contenido en ceniza depende del propio origen de la biomasa donde el incremento de cenizas en el biocombustible es perjudicial en proceso de combustión o en cualquier otro proceso termoquímico, pues se tiende a incrementar la formación de escorias dentro de las calderas (Fernández, 2011).

\subsection{Humedad}

Con lo referente al contenido de humedad se alcanzó el $11.98 \%$ en la mezcla 3 (cascarilla 4\%, polvillo $14 \%$, cuesco $10 \%$ y aserrín $14 \%$ ), este valor es similar al $12.3 \%$ señalados por (Gallipoliti, V; Martina, P; Corace , J; Aeberhardt, R; Garcia, Sola, 2012), En su investigación sobre Fabricación de briquetas con aserrín blanco de pino. Análisis inmediato y obtención de su poder calorífico la cual indica que la humedad del biocombustible sólido es muy importante, porque el grado de humedad de la briqueta depende directamente de su rendimiento, en caso de obtener un mayor porcentaje la misma humedad deformaría la briqueta ya que esta no lleva ningún tipo de producto químico ni aditivo para su compactación
(Gallipoliti, V; Martina, P; Corace , J; Aeberhardt, R; Garcia, Sola, 2012).

La forma cuadrada obtuvo $12.78 \%$ y la cilíndrica $12.71 \%$ de humedad, los mismos que están por debajo del dato citado por (Carrillo, y otros, 2014), en su investigación con el tema, "Contenido de humedad y sustancias inorgánicas en subproductos maderables de pino para su uso en pélets y briquetas", en el cual indica que los valores límites permitidos de contenido de humedad $(\mathrm{CH})$ es el $18 \%$.

Con relación al tamaño de la briqueta la humedad presentó un valor alto $(12.63 \%)$ en $c_{0}=10 \mathrm{~cm}$, mientras que: $c_{1}=15 \mathrm{~cm}$ el valor fue inferior (12.36\%), los mismos que están entre los rangos citados por (Carrillo, y otros, 2014), en su investigación con el tema, Contenido de humedad y sustancias inorgánicas en subproductos maderables de pino para su uso en pélets y briquetas, donde indica los valores límites permitidos de contenido de humedad $(\mathrm{CH}) 18 \%$. La forma más sencilla de saber que una briqueta tiene un porcentaje de humedad adecuado es observar su forma, si su forma es completamente rectangular o cuadrada con la mezcla bien compactada la briqueta está en su punto óptimo de humedad caso contrario si se observa que el bloque no está muy compactado y se rompe con gran facilidad significa que la briqueta tiene un porcentaje de humedad demasiado alto (Ortiz, 2008).

El autor (Ortiz, 2008) en su investigación con el tema Producción de biocombustibles sólidos de alta densidad en España señala que es preciso que el contenido de humedad no supere valores del orden del $15 \%$ y que esté por encima 
de valores del $8 \%$, puesto que por debajo de este nivel no se obtienen una adecuada aglomeración, ya que el agua en determinadas proporciones actúa como sustancia termoplástica favoreciendo la autoglomeración $\mathrm{y}$, por tanto, si no alcanzan estos porcentajes mínimos la compactación es defectuosa (Ortiz, 2008).

\subsection{Poder calorífico}

De acuerdo al poder calorífico se alcanzó un valor de 4902,18 Kcal/kg en la mezcla 2 (cascarilla $2 \%$, polvillo $14 \%$, cuesco $12 \%$ y aserrín $12 \%$ ), este valor es similar a los datos reportados por (Gallipoliti, V; Martina, P; Corace , J; Aeberhardt, R; Garcia, Sola, 2012), en su investigación sobre Fabricación de briquetas con aserrín blanco de pino. Análisis inmediato y obtención de su poder calorífico, menciona $4289,4 \mathrm{Kcal} / \mathrm{kg}$ en las briquetas.

El poder calorífico energía en las briquetas de formas cuadradas con valores $5069,08 \mathrm{Kcal} / \mathrm{kg}$, este dato es similar $5092,5 \mathrm{kcal} / \mathrm{kg}$, planteado por los autores (Soto \& Núñez, 2008), en su investigación con el tema "Fabricación de pellets de carbonilla, usando aserrín de pinus radiata (d. don)", esta variación muestra que utilizar diferentes formas para la elaboración de las briquetas influye en su poder calorífico, lo cual, es un aspecto muy importante ya que las briquetas con un alto poder calorífico consiguen elevar la temperatura muy rápidamente (Soto \& Núñez, 2008).

El autor García, (2014). En su investigación con el tema Diseño de proceso y de planta piloto para la fabricación de briquetas de aserrín, señala que el poder calorífico es uno de los aspectos más importantes porqué es la cantidad de energía que desprende el combustible al producirse la combustión ya que altos poderes caloríficos indican un biocombustible de calidad y bajos poderes caloríficos señalan malos combustibles, por lo tanto, se obtiene altos poderes caloríficos ya que las briquetas están elaboradas con subproductos con alto nivel de energía (García, 2014).

\section{CONCLUSIONES}

La Mezcla 3 $\mathrm{a}_{2}$ compuesta de (cascarilla de arroz 4\%, polvillo $14 \%$, cuesco $10 \%$, aserrín $12 \%$ y $60 \%$ de agua), obtuvo diferencia significativa en cenizas con el $4.96 \%, 11.98 \%$ humedad y un poder calorífico de $4917.43 \mathrm{kcal} / \mathrm{kg}$, por lo tanto, se acepta la hipótesis alternativa y se concluye que las concentraciones de los diferentes desechos Agroindustriales influyen en el proceso de elaboración de briquetas como biocombustible.

Se observó diferencia significativa en las briquetas de formas cuadradas, por lo tanto, se acepta la hipótesis alternativa y se concluye que la utilización de diferentes formas en la elaboración de las briquetas afecta la calidad $y$ el rendimiento energético.

De acuerdo a los resultados obtenidos el tamaño óptimo de la briqueta fue la de $15 \mathrm{~cm}=c_{1}$ ya que presento diferencia significativa en humedad con el (12.63\%), y un elevado poder calorífico de (5025.06 $\mathrm{kcal} / \mathrm{kg}$ ), por lo tanto, se acepta la hipótesis alternativa y se concluye que las características físicas varían en las briquetas elaboradas a partir de residuos Agroindustriales. 
Tabla 3. Costo de materias primas directas en el proceso de elaboración de las briquetas.

\begin{tabular}{|c|c|c|c|c|c|c|c|c|c|c|c|c|c|}
\hline \multirow{2}{*}{ INSUMOS } & \multirow[b]{2}{*}{ CANTIDAD (g) } & \multicolumn{4}{|c|}{ Mezcla 1} & \multicolumn{4}{|c|}{ Mezcla 2} & \multicolumn{4}{|c|}{ Mezcla 3} \\
\hline & & $a_{0} b_{0} c_{0}$ & & $a_{0} b_{1} c_{0}$ & $a_{0} b_{1} c_{1}$ & $a_{1} b_{0} c_{0}$ & $a_{1} b_{0} c_{1}$ & $a_{1} b_{1} c_{0}$ & $a_{1} b_{1} c_{1}$ & $a_{2} b_{0} c_{0}$ & $a_{2} b_{0} c_{1}$ & $a_{2} b_{1} c_{0}$ & $\overline{a_{2} b_{1} c_{1}}$ \\
\hline C. de Arroz & $50-100-200$ & 0.003 & 0.003 & 0.003 & 0.003 & 0.005 & 0.005 & 0.005 & 0.005 & 0.050 & 0.050 & 0.050 & 0.050 \\
\hline Polvillo & $800-700-700$ & 0.045 & 0.045 & 0.045 & 0.045 & 0.040 & 0.040 & 0.040 & 0.040 & 0.040 & 0.040 & 0.040 & 0.040 \\
\hline Cuesco & $400-600-500$ & 0.060 & 0.060 & 0.060 & 0.060 & 0.025 & 0.025 & 0.025 & 0.025 & 0.080 & 0.080 & 0.080 & 0.080 \\
\hline Aserrín & $750-600-600$ & 0.048 & 0.048 & 0.048 & 0.048 & 0.038 & 0.038 & 0.038 & 0.038 & 0.038 & 0.038 & 0.038 & 0.038 \\
\hline Agua & $3000-3000-3000$ & 0.025 & 0.025 & 0.025 & 0.025 & 0.025 & 0.025 & 0.025 & 0.025 & 0.025 & 0.025 & 0.025 & 0.025 \\
\hline \multicolumn{2}{|c|}{ Total, costo fijo } & 0.181 & 0.181 & 0.181 & 0.181 & 0.133 & 0.133 & 0.133 & 0.133 & 0.233 & 0.233 & 0.233 & 0.233 \\
\hline \multicolumn{2}{|c|}{ \% Rendimiento } & 0.188 & 0.440 & 0.253 & 0.510 & 0.165 & 0.318 & 0.206 & 0.404 & 0.253 & 0.488 & 0.257 & 0.301 \\
\hline \multicolumn{2}{|c|}{ Conversión a briqueta kg } & 0.235 & 0.549 & 0.316 & 0.638 & 0.206 & 0.397 & 0.258 & 0.505 & 0.316 & 0.609 & 0.321 & 0.576 \\
\hline \multicolumn{2}{|c|}{ Costo de producción USD por kg } & 0.768 & 0.329 & 0.571 & 0.283 & 0.646 & 0.335 & 0.516 & 0.263 & 0.737 & 0.383 & 0.726 & 0.405 \\
\hline
\end{tabular}

\section{REFERENCIAS}

[1]. Carrillo, A., Correa, F., Rutiaga, J., Márquez, F., González, Jurado, E., \& Garza, F. (2014). Contenido de Humedad y Sustancias Inorgánicas en Subproductos Maderables de Pino. Chapingo Serie Ciencias Forestales y del Ambiente (Scielo).

[2]. Cediel, Alexandra; Rivera, José L; Montalvo, Andrea S; Sierra, Fabio E; Forero, Carlos, «Estudio preliminar del potencial energético de cuesco de palma y cáscara de coco en Colombia,» Ingeniería Solidaría, Vol. 8, No 14, P. 20, 2012.

[3]. Da Silva, D. (2013). Proyecto de Creacion de una Fabrica de
Briquetas de Aserrin en Santa Rosa del Aguaray. Santa Rosa- Praguay.

[4]. Fernández Llorente, $M$. (2011). Caracteristicas, barreras y retos paara la implementación comercial de la biomasa sólida como combustible. Vida Rural, 60 - 64.

[5]. Fernández, M. (2011). Caracteristicas, barreras y retos para la Implementación comercial de la Biomasa Sólida como Combustible. Vida Rural, 60 - 64.

[6]. Forero Núñez, Carlos Andrés; Guerrero Fajardo, Carlos Alberto; Sierra Vargas, Fabio Emiro; «Producción y Uso de Pellets de Biomasa para La Generación de 
Energía Térmica: Una Revisión A Los Modelos Del Proceso De Gasificación,» Iteckne, Vol. 9, No 1 , P. 22, 2012.

[7]. Gallipoliti, V; Martina, P; Corace , J; Aeberhardt, R; Garcia, Sola. (2012). FABRICACION DE BRIQUETAS CON ASERRIN BLANCO DE PINO. ANALISIS INMEDIATO Y OBTENCION DE SU PODER CALORIFICO. 10(2), 129-137.

[8]. García, M. (2014). Diseño de Proceso y de Planta Piloto para la Fabricación de Briquetas de Aserrín,. Perú.

[9]. Mera, M; Simbaña, E, «Evaluación de la capacidad calorífica de biocombustible sólido a partir de residuos lignocelulósicos de café (Coffea Spp) frente a leña de Espino (Vachellia Macracantha) y eucalipto (Eucalyptus Globulus Labill),» Axioma, N 15, P. 36, 2016.

[10]. Mosquera, P., \& Merino, L. (2006). Eficiencia Energética y Kioto de Empresa y Energías Renovables. Fundación confemetal, 184.

[11]. Muñoz Muñoz, D., Pantoja, M., Alvaro, J., Cuatin, G., \& F., I. (2014). Aprovechamiento de residuos Agroindustriales como combustible. Biotecnología en el sector agropecuario, 12(2), 20.

[12]. Ortiz, L. (2008). Producción de Biocombustibles Sólidos de Alta densidad en España (Vol. 5). España: Gamesal.

[13]. Salinas, E; Gasca, Víctor., "Los Biocombustibles," El Cotidiano, No 157, P. 76, 2009.

[14]. Soto, G., \& Núñez, M. (2008). Fabricación de Pelletsde Carbonilla usando aserrín de Pinus Radiata , como material Aglomerante. Maderas, Ciencia Y Tecnología., 10(2), 129-137.

[15]. Valderrama, A., Curo, H., Quispe, C., Llantoy, V., \& Gallo, J. (2014). Briquetas de Residuos Sólidos orgánicos como Fuente de Energía Calorífica en cocinas no Convencionales. Centro de Desarrollo e Investigación en Termofluidos CEDIT . Recuperado el 20020, de Centro de Desarrollo e Investigación en Termofluidos CEDIT .

[16]. Vargas, J., Alvarado, P., Vega-Baudrit, J., Porras M, «Caracterización del subproducto cascarilla de arroz en búsquedas de posibles aplicaciones como materia prima en procesos.," Dialnet, Vol. 23, No 1, P. 91, 2013. 
solid biofuel from waste generated by agroindustrial 